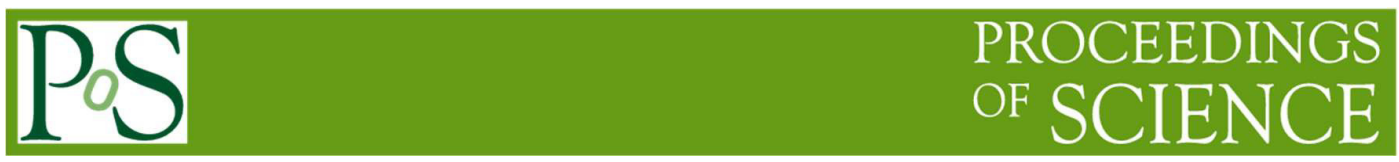

\title{
Experiment on search for $n$-nbar oscillations using a projected UCN source at the WWR-M reactor
}

\author{
Alexey Fomin ${ }^{1}$ \\ Petersburg Nuclear Physics Institute NRC KI \\ Gatchina, Russia \\ E-mail: fomin akepnpi.nrcki.ru
}

In Petersburg Nuclear Physics Institute (PNPI) a project of the source of ultracold neutrons (UCN) based on superfluid helium for the WWR-M reactor has been developed. It must provide UCN density 2-3 orders of magnitude higher than existing sources. For the new source we propose an experiment on search for neutron-antineutron oscillations based on the storage of ultracold neutrons in a material trap. The sensitivity of the experiment mostly depends on the trap size and the amount of UCN in it. The results of simulations of the designed experimental scheme show that the sensitivity can be increased by $\sim 10-40$ times compared to sensitivity of previous experiment depending on the model of neutron reflection from walls.

The 26th International Nuclear Physics Conference

11-16 September, 2016

Adelaide, Australia

\section{${ }^{1}$ Speaker}




\section{Introduction}

Modern physics has a big interest in search for neutron-antineutron oscillations in connection with question of existence of baryon number violation [1]. Firstly, it was suggested to search for neutron-antineutron oscillations in beam experiments [2]. In this experiment beam of neutrons after long flight base hits target where annihilation reaction is searched. All flight base should have shielding from the Earth's magnetic field because it suppresses amplitude of oscillations. Such experiment was realized at beam of cold neutrons at Institute Laue-Langevin (ILL) [3,4]. In this experiment current limit on period of neutron-antineutron oscillations of $8.6 \cdot 10^{7} \mathrm{~s}$ was obtained. Sensitivity of experiment depends on so called discovery potential $N t^{2}$, where $N$ is number of neutrons reaching the target per second and $t$ is time of neutron flight to the target. Discovery potential of ILL experiment was $1.5 \cdot 10^{9} \mathrm{n} \mathrm{s}$.

Later it was suggested experimental scheme with storage of ultracold neutrons (UCN) in a material trap [5]. UCN with wavelengths about $1000 \AA$ have unique feature: they are totally reflected from the surface of many materials under any angle of incidence. This effect allows storage of UCN in material traps and transporting along pipes (neutron guides). Due to its properties UCN are widely used in experimental research [6]. In the case of experiment on search for neutronantineutron oscillations the walls of UCN trap play the role of annihilation target. The sensitivity of the experiment mostly depends on the trap size and the amount of UCN in it.

Progress can be reached due to supersource of UCN on the basis of superfluid helium which is under construction now in PNPI [7]. This source is expected to yield UCN density of $10^{4} \mathrm{~cm}^{-3}$, i.e., approximately two-three orders of magnitude higher than the density from existing UCN sources in the world [8]. UCN production by the source will be about $10^{8} \mathrm{n} / \mathrm{s}$. We propose to search for neutron-antineutron oscillations in experiment at the new UCN source.

\section{Sensitivity of experiment}

In our work [9] we estimated possible sensitivity of experiment on search for neutron-antineutron oscillations at the new UCN source. We used two models of neutron reflection from trap walls: with partial accumulation of the antineutron phase and without it. Real parts of the reflection potential are close or coinciding for the case with partial accumulation of the antineutron phase. For the case without accumulation - real part of the reflection potential for antineutron is close to zero. In the first case, one expects antineutrons to reflect from walls and the antineutron phase to be accumulated in contrast to the second case, in which no such accumulation can take place because the antineutrons immediately annihilate upon entering the matter. However, the coefficient of antineutron reflection in the first case cannot be sufficiently high because of a large imaginary part of the reflection potential for antineutron due to a large annihilation cross section. Calculations showed that for the trap in form of vertical cylinder with radius of $3 \mathrm{~m}$ and height of $2.5 \mathrm{~m}$ ILL sensitivity level can be increased by $\sim 20-80$ times depending on the model of neutron reflection from walls.

At the planning stage we realized that the trap size should be reduced in order to fit for available space in reactor hall. The appropriate area for UCN trap cannot exceed $2 \times 4 \mathrm{~m}$. Smaller trap size reduces the sensitivity of the experiment. It has been estimated using the Monte Carlo method like in previous work. UCN are supplied from a source with superfluid helium to the experimental trap in a steady-state regime via a neutron guide of 140-mm diameter. The trap walls are coated 
by Be with boundary velocity of $6.8 \mathrm{~m} / \mathrm{s}$ and a loss factor of $3 \cdot 10^{-5} \cdot 90 \%$ of neutron reflections from the trap walls are diffuse. Efficiency of annihilation events detection was not considered in this work. According to available area we accepted UCN trap in form of horizontal cylinder. Length of the trap is $4 \mathrm{~m}$. Time of neutron flight distribution is shown in figure 1. Sensitivity of experiment dependence on diameter of UCN trap is shown in figure 2.

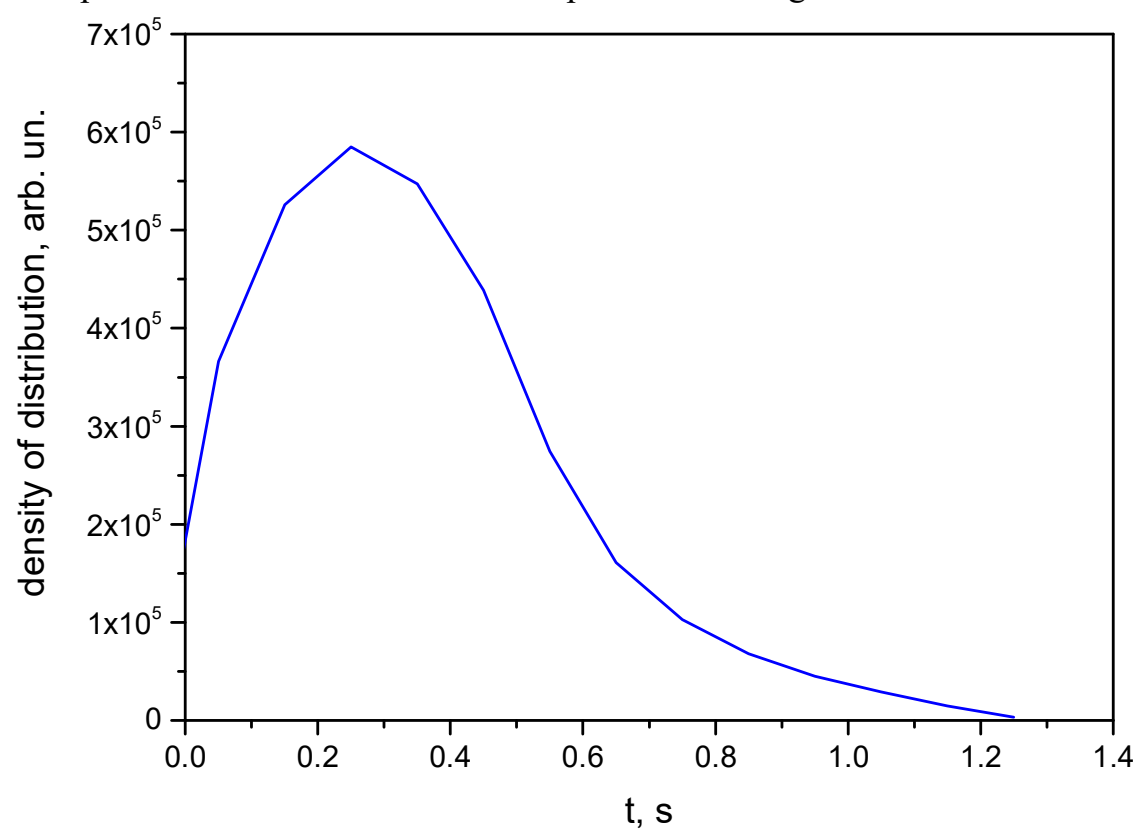

Figure 1. Time of neutron flight distribution for the trap in form of horizontal cylinder with diameter of $2 \mathrm{~m}$. Length of the trap is $4 \mathrm{~m}$.

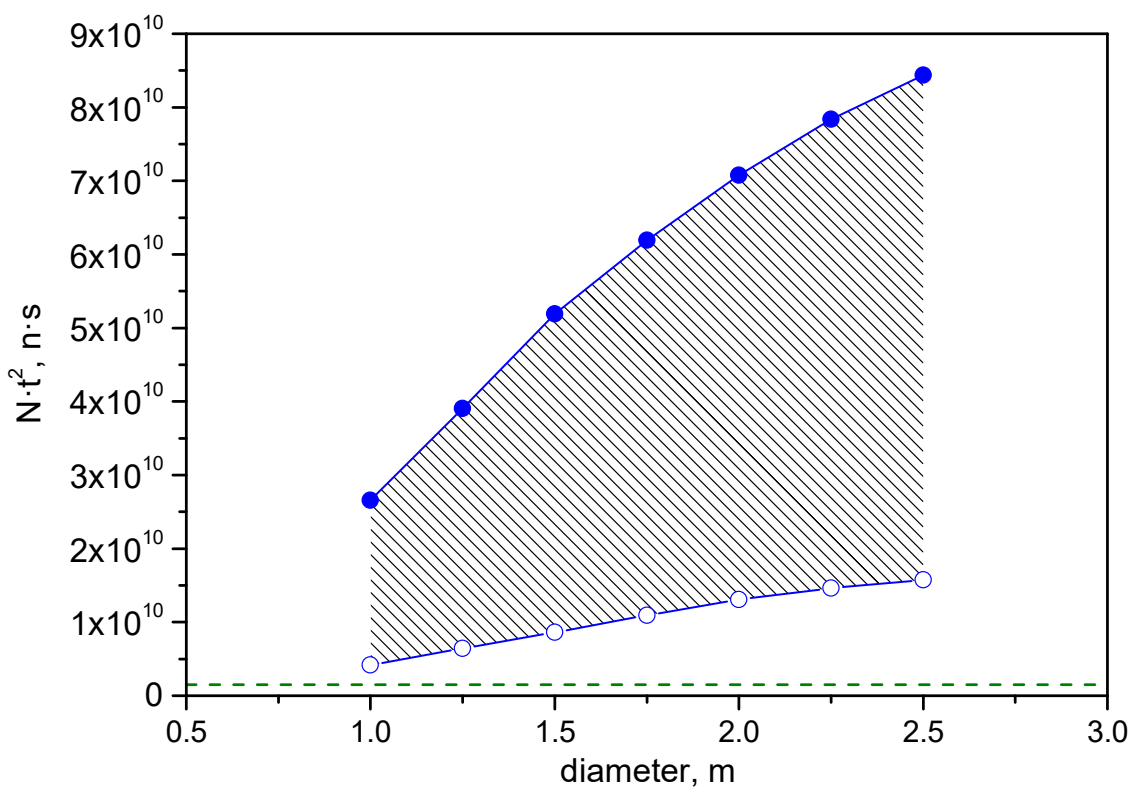

Figure 2. Sensitivity of experiment depending on diameter of UCN trap. Length of the trap is $4 \mathrm{~m}$. Filled signs correspond to the case with partial accumulation of the antineutron phase. Empty signs correspond to the case without accumulation of the antineutron phase. Dashed horizontal line corresponds to sensitivity of the ILL experiment. 
So we can obtain increase to ILL sensitivity level by $\sim 10-40$ times for the trap in form of horizontal cylinder with diameter of $2 \mathrm{~m}$ and length of $4 \mathrm{~m}$. The period of oscillations is determined as $\tau_{n \tilde{n}}=\sqrt{\frac{\left(N \cdot t^{2}\right) \cdot T \cdot \varepsilon}{\tilde{N}}}$, where $T$ is the time of statistics accumulation, $\varepsilon$ is the efficiency of detection, and $\tilde{N}$ is the number of candidate events. In the absence of antineutron events ( $\tilde{N}$ $\leq 2.3$ at $90 \% \mathrm{CL}$ ) for a 3 -year period of time at $\varepsilon=0.9$, it is possible to obtain limit on the oscillation period on a level of $\tau_{n \tilde{n}} \geq(0.7-1.4) \cdot 10^{9} \mathrm{~s}(90 \% \mathrm{CL})$.

In concluding, it should be mentioned that there is a project of an experiment on search for neutron-antineutron oscillations with cold neutrons on the European Spallation Source (ESS) [1]. It is planned to increase the sensitivity by two to three orders of magnitude as compared to that in the ILL experiment, which would be about order of magnitude better than in the proposed variant with UCN storage trap. However, it is important to obtain result in an independent experiment using an alternative method. Comparison from financial point of view for different projects is also interesting, because realization of experiment with UCN is much more compact.

\section{Design of the setup}

The scheme of the setup is shown in figure 3. The detection technic is similar to the technic of the ILL experiment $[3,4]$. UCN in the experiment are stored in the trap (1), which is a cooper cylinder with length of $4 \mathrm{~m}$ and diameter of $2 \mathrm{~m}$. UCN are transported to the trap by the neutron guide through hole in a side of the cylinder. The UCN trap is mounted in an aluminum vacuum chamber (2). Pumping the vacuum chamber is implemented through a pipe of $200 \mathrm{~mm}$ in an upper side of the setup. For suppress the Earth's magnetic field the vacuum chamber is surrounded by magnetic shield (3). To reconstruct the position where annihilation takes place the vertex detector (4) is used. The calorimeter (5) is used to reconstruct energies of annihilation reaction products. Design of experimental setup is shown in figure 4.

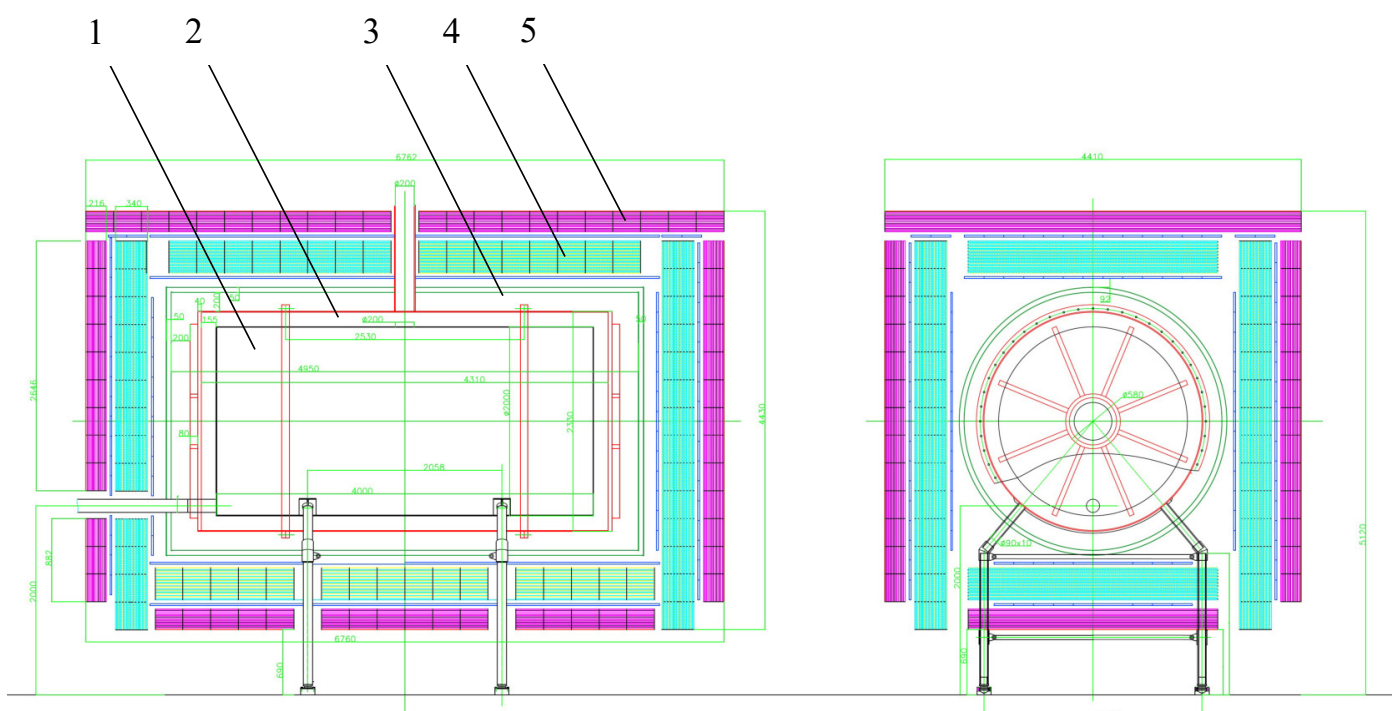

Figure 3. General scheme of experimental setup: 1 - UCN trap, 2 - vacuum chamber, 3 - magnetic shield, 4 - trek detector, 5 - calorimeter. 


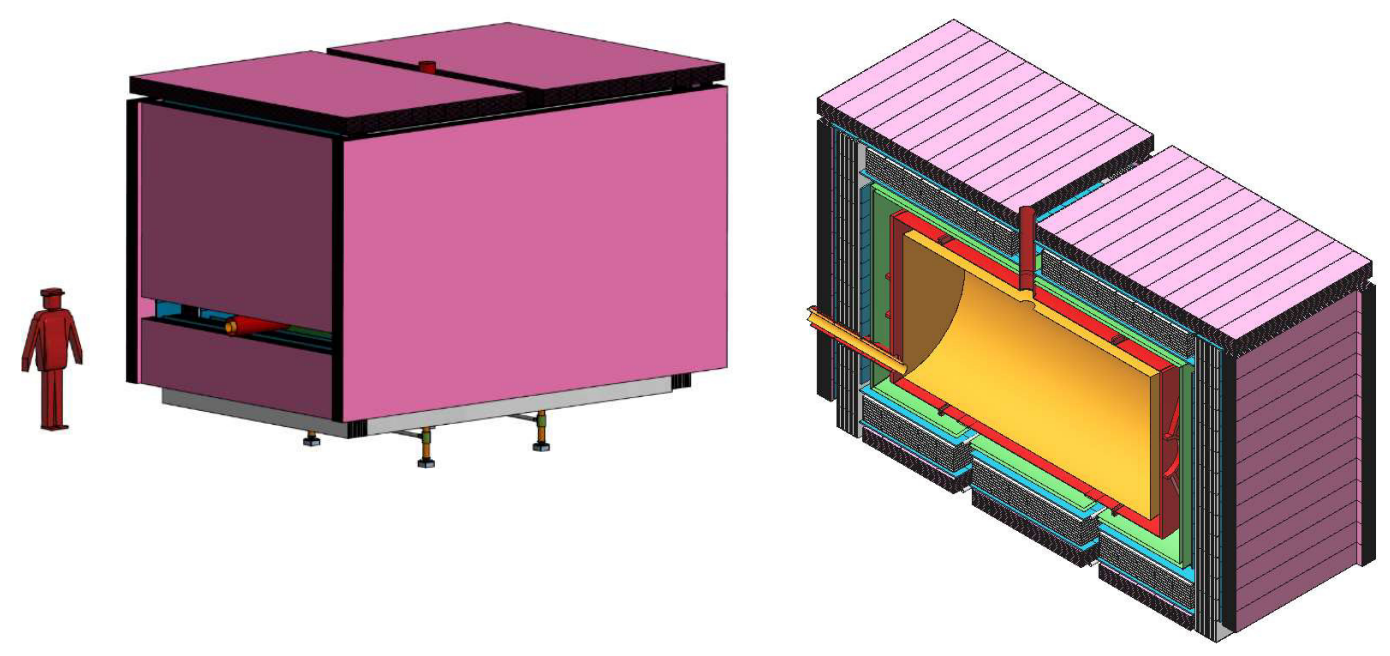

Figure 4. Design of experimental setup.

\section{Acknowledgments}

This work was supported by the Russian Foundation for Basic Research, grant no. 16-02-00778-a.

\section{References}

[1] D.G. Phillips II, W.M. Snow, K. Babu et al. 2016 Physics Reports 6121

[2] M.V. Kazarnovskii V.A. Kuz'min, K.G. Chetyrkin, M.E. Shaposhnikov, 1980 JETP Lett. 3282

[3] M. Baldo-Ceolin, P. Benetti, T. Bitter et al. 1990 Phys. Lett. B 23695

[4] M. Baldo-Ceolin, P. Benetti, T. Bitter et al. 1994 Z. Phys. C 63409

[5] M.V. Kazarnovskii, V.A. Kuz'min, M.E. Shaposhnikov 1981 JETP Lett. 3447

[6] A.P. Serebrov 2015 Physics-Uspekhi 581074

[7] A.P. Serebrov 2011 Crystallogr. Rep. 561230

[8] A.P. Serebrov, A.K. Fomin 2015 Tech. Phys. 601238

[9] A.P. Serebrov, A.K. Fomin, Yu.A. Kamyshkov 2016 Tech. Phys. Lett. 4299 